\title{
Explanation of Dark Matter, Dark Energy and Dark Space: Discovery of Invisible Universes
}

\author{
Alexander Alexandrovich Antonov \\ Independent Researcher, Kiev, Ukraine \\ Email: telan@bk.ru
}

How to cite this paper: Antonov, A.A. (2019) Explanation of Dark Matter, Dark Energy and Dark Space: Discovery of Invisible Universes. Journal of Modern Physics, 10, 1006-1028. https://doi.org/10.4236/jmp.2019.108067

Received: June 4, 2019

Accepted: July 22, 2019

Published: July 25, 2019

Copyright $\odot 2019$ by author(s) and Scientific Research Publishing Inc. This work is licensed under the Creative Commons Attribution International License (CC BY 4.0).

http://creativecommons.org/licenses/by/4.0/

\begin{abstract}
Theoretical and experimental studies of special processes in linear electric circuits have proved the principle of physical reality of imaginary numbers discovered 500 years ago. This principle of physical reality of imaginary numbers has allowed astrophysics to prove the existence of invisible parallel universes and explain the phenomena of dark matter, dark energy and dark space associated therewith. Physical entities corresponding to imaginary, complex and hypercomplex numbers in the other exact sciences together form an invisible and still unknown world that is to be learned by science of the future.
\end{abstract}

\section{Keywords}

Imaginary Numbers, Dark Matter, Dark Energy, Dark Space, Multiverse, Hyperverse

\section{Introduction}

At the turn of the twentieth century William Thomson, 1st Baron Kelvin the President of the Royal Society in 1890-1895, asserted: "There is nothing new to be discovered in physics now. All that remains is more and more precise measurement." However, the twentieth century denied this assertion, as theory of relativity and quantum mechanics were developed, radioactivity and X-ray radiation, a planetary model of atom and black holes, dark matter and dark energy were discovered, atomic and hydrogen bombs were exploded, transistors, integrated microcircuit, lasers and many other things were created.

History repeats itself. And now, at the turn of the twenty-first century, it is often asserted that we just need to create a "theory of everything", thereby allowing one to assume that everything else in physics has already been learned ${ }^{1}$.

But, firstly, it is more correct to assume that the learning process is infinite ${ }^{1}$ Therefore, unified field theory is more correct name of this theory. 
and there are always more unlearned things in physics than learned ones. Therefore, for example, the achievements of twentieth-century physics from the standpoint of physics of the third millennium would be much more modest than we imagine today. Secondly, many theories developed in physics up to date, including the theory of relativity and quantum mechanics, are not actually completely accurate and require correction in accordance with the principle of physical reality of imaginary numbers.

Finally, authors of a number of fundamental discoveries have been honoured with the Nobel Prize already in the twenty-first century:

- 2006: John C. Mather and George F. Smoot, who confirmed the Big Bang theory by their studies of cosmic microwave background and black holes;

- 2011: Saul Perlmutter, Brian P. Schmidt and Adam G. Riess, who discovered dark energy;

- 2013: François Englert and Peter Higgs, who discovered the Higgs boson.

There can be no doubt that there will be other outstanding discoveries and new theories in the present century.

In this regard, the hypothesis of the hidden Multiverse developed in the twenty-first century is also of interest, as it has solved many problems that arose in astrophysics of the twentieth century. This hypothesis is based on the principle of physical reality of imaginary numbers proved theoretically and experimentally. Moreover, there are even several proofs [1]-[17] that have been offered. And since previously unknown experiments ${ }^{2}$ corresponding to them can be repeated in any radio engineering and electrotechnical laboratory, they, unlike the unsuccessful OPERA experiment, which attempted to solve the same problem essentially, are absolutely reliable and undoubtedly demonstrative.

The simplest and most understandable of these proofs is the proof using the Ohm's law in the interpretation of Steinmetz [10] [11] [12] [13] [14]. This interpretation of the Ohm's law, in addition to its direct purpose of enabling calculation of linear electric AC circuits, also has allowed the author to:

- prove physical reality of imaginary and complex numbers ${ }^{3}$ [1]-[17];

- create correct theory of resonance [1] [2] [3] [4] [7] [9] [15] [16];

- correct the special theory of relativity (STR) [7] [10] [13] [18];

- create verifiable hypothesis of the hidden Multiverse [19]-[34];

- explain the phenomenon of dark matter and dark energy [8] [11] [14] [17] [23]-[30] [35] [36] [37] [38];

- explain where antimatter is and why it does not annihilate with matter [38];

- explain where tachyons are and why they do not violate the causality principle [38];

- discover the phenomenon of dark space and a Hyperverse [17] [39].

${ }^{2}$ Including those described in articles [1] [2] [3] [5], published before the end of the OPERA experiment and therefore made this experiment unnecessary.

${ }^{3}$ Physical reality of imaginary, complex, hyper-complex and real numbers is actually reasonable to speak of only in respect of concrete numbers provided with references to units of measurement used for the corresponding parameters of physical objects. 


\section{Proof of Physical Reality of Concrete Imaginary Numbers}

In 1826 Georg Simon Ohm discovered the law, which now bears his name, as a result of nine years of experimental research. In order to realize how difficult it was to discover the law, it's enough to remember that there were no electric measuring instruments at that time. Moreover, experimental research and mathematical processing of its results were not encouraged in physics of that time. Famous physicist Alexander Grigorievich Stoletov wrote in this regard: “...physics especially tempted natural philosophers. What a favourable theme were electric phenomena for the most riotous imaginations... Attractive and vague deductions were in the foreground: hard work of experimenter and exact mathematical analysis were not honoured; they seemed superfluous and harmful in the study of nature..." Therefore, in $1828 \mathrm{Ohm}$ was even fired by the Minister of Education for his discovery. The Minister considered that the use of mathematics in physics was inappropriate. However, later, Ohm's research received deserved recognition. In 1881 International Congress of Electricians in Paris gave his name to the unit of electric resistance.

Nevertheless, exceptional ideological significance of Ohm's law for all exact sciences has not been still realized. Therefore, except solution of the above-specified problems, this article aims to fill this gap in science and pay tribute to the great scientist.

In 1826 Georg Simon Ohm proposed his law in the formulation applicable to electric DC circuits [40]. And its interpretation applicable to electric AC circuits was proposed by Charles Proteus Steinmetz [41] in 1893. This interpretation is now commonly referred to as the symbolic method of calculating electric circuits. We will use it below.

According to Steinmetz theory resistance of a resistor is equal to a real number $R$, the value of which does not depend on frequency $\omega$ of sinusoidal voltage applied to thereto. Inductive reactance $L$ is equal to a positive imaginary number $^{4} j \omega L$, the value of which depends on frequency $\omega$. Capacitive reactance $C$ is equal to a negative imaginary number $-j / \omega C$, the value of which also depends on frequency $\omega$, although in a different way.

Consequently, resistance of any electric $L C R$ circuit would depend on frequency $\omega$, if imaginary inductive and capacitive reactances are actually physically existent and would not depend, if otherwise. Therefore, an experiment determining whether inductive and capacitive imaginary reactances are physically existent would be extremely simple. In this experiment, it is only needed to change the frequency of sinusoidal voltage applied to $L C R$ circuit and check whether value of electric current flowing through the circuit changes. The result of the experiment conducted by millions of engineers in the course of their everyday practical activities (for example, when creating electric filters that are indispensible for radio engineering, telecommunication, television, radiolocation ${ }^{4}$ In the theory of electric circuits, unlike mathematics, the imaginary unit $\sqrt{-1}$ is usually denoted by the letter $j$, since the letter $i$ denotes electric current. 
and other exact sciences) has long been known. In this experiment intensity of current always changes.

Besides, physical reality of imaginary inductive and capacitive reactances is also confirmed by the power factor, also called the $\cos \varphi$, a concept available in power engineering, characterizing the presence in the load of a physically existent imaginary component of the power consumed.

Consequently, imaginary inductive and capacitive reactances are actually physically existent. Hence, any other concrete imaginary numbers are also physically existent.

The utmost importance of imaginary numbers in the science was emphasized by Sir Roger Penrose: “...the very system of complex numbers has a profound and timeless reality which goes beyond the mental constructions of any particular mathematician... They were put there neither by Cardano, nor by Bombelly, nor Wallis, nor Coates, nor Euler, nor Wessel, nor Gauss, despite the undoubted farsightedness of these, and other, great mathematicians; such magic was inherent in the very structure that they gradually uncovered..."

Discovery of the experimentally proven principle of physical reality of imaginary numbers is extremely important from ideological point of view. The principle suggests that in addition to the visible world known to us there is another world which is invisible and still unknown. And, as shown below, under certain circumstances people can visit this unknown physical world even passing into it on foot.

Thus, Ohm's law is important in science because it allows calculation of electric circuits, and even more important because it allows proof of existence of the invisible and unknown world, which the science of future has yet to discover. As well as because, in accordance with the principle of physical reality of imaginary numbers, all existing theories and hypotheses in exact sciences should now be corrected. Their existing interpretations, as shown below with the example of the currently accepted version of the special theory of relativity, are imperfect.

\section{Correction of the Special Theory of Relativity}

The special theory of relativity [42] [43] [44] created by Joseph Larmor [45], Nobel laureate Hendrik Antoon Lorentz [46], Jules Henri Poincaré [47], Nobel laureate Albert Einstein [48] and other outstanding scientists has always been criticized throughout its century-old history. The criticism ${ }^{5}$ has not always been constructive, although sometimes it has been fair and incontestable. For example, the principle of non-exceedance of the speed of light postulated in the STR, ${ }^{5}$ The STR has been criticized from the very beginning by Oliver Heaviside, Nikola Tesla, Nobel Prize winner Albert Abraham Michelson, Nobel Prize winner Friedrich Wilhelm Ostwald, Nobel Prize winner Joseph Noble Prize winner Nobel Prize winner Svante August Arrhenius, Nobel Prize winner Prize winner Alvar Gullstrand, Nobel Prize winner Wilhelm Carl Werner Otto Fritz Franz Wien, Nobel Prize winner Walter Hermann Nernst, Nobel Prize winner Ernest Rutherford, 1st Baron Rutherford of Nelson, Nobel Prize winner Johannes Stark, Nobel Prize winner Frederick Soddy, Nobel Prize winner Percy Williams Bridgman, Nobel Prize winner Edwin Mattison McMillan, Nobel Prize winner Hideki Yukawa, Nobel Prize winner Hannes Olof Gösta Alfven and many other outstanding scientists.

${ }^{6}$ As it has not been yet proved by anybody. 
which has the effect of denying the physical reality of imaginary numbers, is experimentally refuted by the principle of physical reality of imaginary numbers proved above.

The Nobel Prize winner Steven Weinberg spoke about the unproven experimental theories very clearly: "Scientific theories cannot be deduced by purely mathematical reasoning".

The principle of physical reality of imaginary numbers also proves incorrectness of all relativistic formulas of the STR, in particular,

$$
\begin{gathered}
m=\frac{m_{0}}{\sqrt{1-(v / c)^{2}}} \\
\Delta t=\Delta t_{0} \sqrt{1-(v / c)^{2}} \\
l=l_{0} \sqrt{1-(v / c)^{2}}
\end{gathered}
$$

where $m_{0}$ is the rest mass of a physical body;

$m$ is the relativistic mass of a moving physical body;

$\Delta t_{0}$ is the rest time of a physical body;

$\Delta t$ is the relativistic time of a moving physical body;

$l_{0}$ is the rest longitudinal length of a physical body;

$l$ is the relativistic longitudinal length of a moving physical body;

$v$ is the velocity of a moving physical body;

$c$ is the speed of light.

Explaining the formulas, the STR asserts that as relativistic mass $m$, time $\Delta t$, longitudinal length $l$ and other physical quantities assumes imaginary values at $v \geq c$, they are physically nonexistent. However, as follows from the principle of physical reality of imaginary numbers, this assertion is erroneous and made due to misunderstanding of some aspects of the STR by its authors, particularly, inability to prove physical reality and explain physical nature of concrete imaginary numbers.

In fact, according to the principle of physical reality of imaginary numbers, physical world corresponding to them exists in the STR, and therefore needs an explanation. This is as follows. When $v \geq c$ physical world must exist if formulas (1), (2) and (3) are valid. But formulas (1), (2) and (3) are valid only in the range $0 \leq v<c$. And in the range $v \geq c$ they do not meet this condition (see Figures 1(a)-(c)). Therefore, they should be corrected for the range $v>c$ as follows

$$
\begin{gathered}
m=\frac{m_{0} i^{q}}{\sqrt{1-(v / c-q)^{2}}}=\frac{m_{0} i^{q}}{\sqrt{1-(w / c)^{2}}} \\
\Delta t=\Delta t_{0} i^{q} \sqrt{1-(v / c-q)^{2}}=\Delta t_{0} i^{q} \sqrt{1-(w / c)^{2}} \\
l=l_{0} i^{q} \sqrt{1-(v / c-q)^{2}}=l_{0} i^{q} \sqrt{1-(w / c)^{2}}
\end{gathered}
$$

where $q=\lfloor v / c\rfloor$ is the "floor" function of argument $v / c$; 


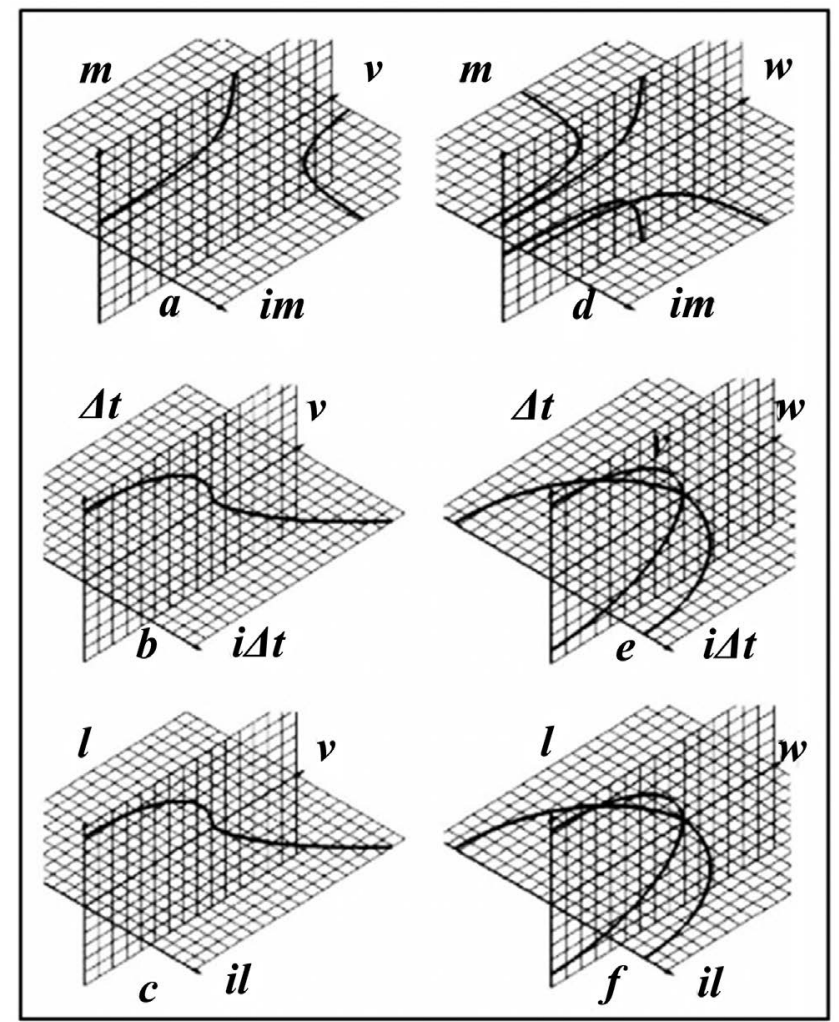

Figure 1. (a) is graph of the function (1); (b) is graph of the function (2); (c) is graph of the function (3); (d) is graph of the function (4); (e) is graph of the function (5); (f) is graph of the function (6).

$w=v-q c$ is the local velocity for each universe, which can take values only in the range $0 \leq w<c$;

$v$ is the velocity measured from our universe;

$c$ is the speed of light.

Albert Einstein did not exclude the adjustment of the STR in future. He wrote: "No single idea, which I would be sure that it will stand the test of time".

\section{Hypothesis of the Hidden Multiverse}

In formulas (4), (5), (6) the value $q=0$ corresponds then to our universe and the value $q=1$ corresponds to the adjacent universe, in which $c \leq v<2 c$ and which is invisible from our universe, as it is beyond the horizon of events. This universe contains tachyons [49]-[54], so it shall be for definiteness referred to as tachyon universe. Recognizing physical reality of imaginary time corresponding to the formula (5), Stephen William Hawking wrote in this regard: "Imaginary time is a new dimension, at right angles to ordinary, real time".

For similar reasons, our universe is called tardyon. Consequently, we actually live in a Multiverse, which shall be called hidden due to mutual invisibility of parallel $^{7}$ universes contained therein, rather than in a Monoverse.

However, in formulas (4), (5), (6) the quantity $q$ can also assume greater val${ }^{7}$ Since despite their infinity they never intersect. 
ues ${ }^{8}$. Thus, the quantity $q=2$ corresponds to the tardyon antiverse (as $i^{2}=-1$ ), the quantity $q=3$ corresponds to the tachyon antiverse (as $i^{3}=-i$ ), the quantity $q=4$ corresponds to another ${ }^{9}$ tardyon universe (as $i^{4}=1$ ), the quantity $q=5$ corresponds to another tachyon universe (as $i^{5}=-1$ ), etc. It can be assumed that these universes can form both closed $^{10}$ (Figure 2) and open (Figure 3, Figure 4) screw structure. Herewith, if the hidden Multiverse has the structure of a closed screw ring ${ }^{11}$, then it should be assumed unique. If the hidden Multiverse has the structure of an open screw ring, then its edges would be connected to other Multiverses: twice with the same Multiverse as in Figure 3, or with two different Multiverses ${ }^{12}$, as in Figure 4. And together they form a Hyperverse.

It's time answer one more obvious question: Why parallel universes do not intersect? There can be only one answer: Because they exist in space that has more than three dimensions. In formulas (4), (5) and (6) this circumstance is taken into account by the parameter $q$, which is the fourth spatial dimension. Consequently, distribution of material contents in such a hidden Multiverse is described by function $f_{q}(x, y, z)+i q$ in which $x, y, z$ are the coordinates of the material contents in the corresponding parallel universe, and $q$ is the coordinate of this universe in the fourth spatial dimension.

Besides, parallel universes naturally do not stand still in this four-dimensional space. They continuously drift and often touch each other in numerous spots or even slightly penetrate into each other. In the places of interpenetration certain transition zones ${ }^{13}$ occur, which are usually referred to as portals ${ }^{14}$ or star gates [19] [20] [21] [29] [30] [31] [55] [56] [57]. In Figures 2-4, these multiple bidirectional portals ${ }^{15}$ are denoted by single two-sided arrows.

\section{Where Are Antimatter and Tachyons?}

The great advantage of the hidden Multiverse hypothesis is that it gives answers to many questions that have not yet been explained in astrophysics.

Thus, it is quite obvious that tachyons are in tachyon universes and antiverses. Herewith, it immediately becomes clear that they do not violate the principle of causality, as they are not in our tardyon universe.

It is no less obvious that antimatter [58] [59] [60] [61] [62] is in antiverses. Besides, tardyon antiverse turns out to exist not only relative to our tardyon universe, but there are other tardyon antiverses relative to other tardyon universes.

${ }^{8}$ This is proved below.

${ }^{9}$ Since there are totally not less than twenty universes in the hidden Multiverse.

${ }^{10}$ Same-type universes and antiverses in all figures are indicated by the same colour combination of frame and fill. Besides, colour combinations of frame and fill of the similar universes and antiverses are mutually opposite.

${ }^{11}$ In fact, as is shown below this structure cannot exist in nature.

${ }^{12}$ In which, therefore, frames are of the same color and fills are of different colors for all Multiverses.

${ }^{13}$ In which the quantity $q$ varies by one from one integer value to another, corresponding to the adjacent parallel universes.

${ }^{14}$ Which have nothing to do with "wormholes" in the general theory of relativity.

${ }^{15}$ Through which the material contents of adjacent universes have been exchanged. 


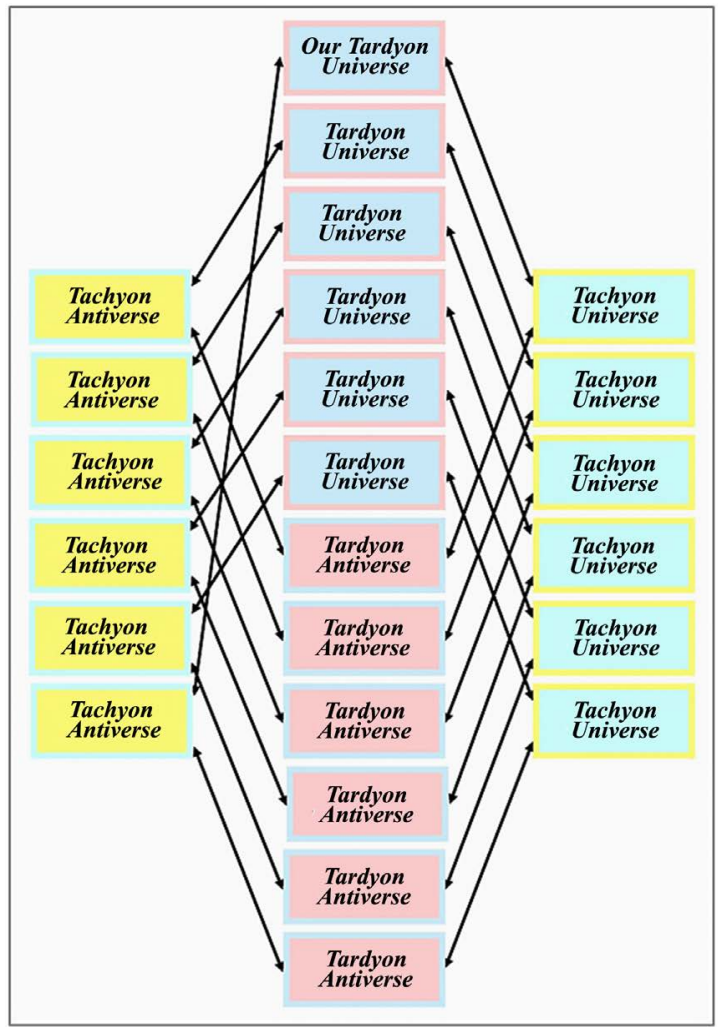

Figure 2. Structure of the hidden Multiverse corresponding to the principle of physical reality of complex numbers.



Figure 3. Another structure of the hidden Multiverse corresponding to the principle of physical reality of complex numbers. 


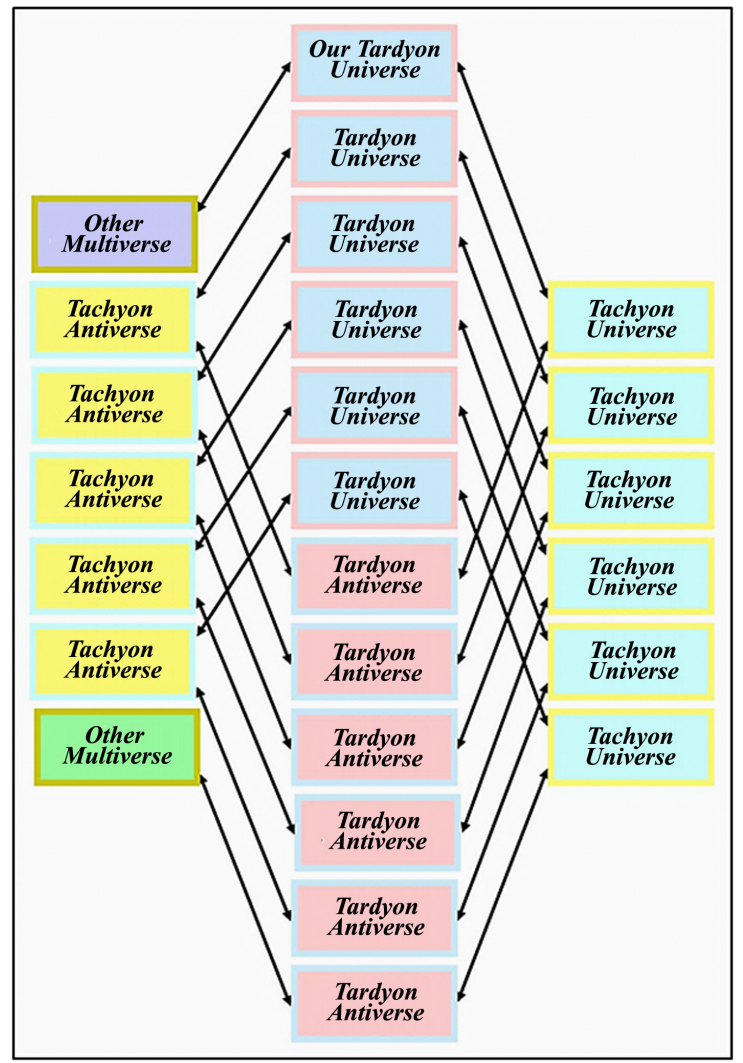

Figure 4. One more structure of the hidden Multiverse corresponding to the principle of physical reality of complex numbers.

Moreover, there are tachyon antiverses relative to their tachyon universes. Therefore, matter and antimatter do not annihilate in the hidden Multiverse, as tardyon universes and antiverses alternate with tachyon universes and antiverses.

\section{Explanation of Dark Matter and Dark Energy}

In the twentieth century two extremely important scientific discoveries were made in astrophysics [63]-[69]. One of them was made in 1932-33 by Jan Hendrik Oort [70] and Fritz Zwicky [71] and called dark matter. Another one, called dark energy, was made in 1998-1999 by Saul Perlmutter [72], Brian P. Schmidt [73] and Adam G. Riess [74] who were awarded the Nobel Prize for this discovery. Adam G. Riess argued on this point: "Humanity is on the verge of a new physics of the Universe ${ }^{16}$. Whether we want it or not, we will have to accept it".

But what dark matter and dark energy themselves are, until very recently, could not be explained. It was for these incomprehensibility that these physical entities were called dark.

Therefore Michio Kaku came to the following conclusion [75]: "Of course, a whole bunch of Nobel Prizes is waiting for the scientists who can reveal the secrets of the 'dark energy' and 'dark matter".

${ }^{16}$ The foundations of which are set forth in this paper. 
There are many hypotheses of the Multiverse [75]-[83]. However, they have a very significant drawback: none of them explains the phenomenon of dark matter and dark energy. The phenomenon of dark matter and dark energy also is not explained by the Monoverse hypothesis corresponding to the current version of the STR, within the framework of which all unsuccessful attempts to explain this phenomenon have been carried out so far. In this regard, it seems appropriate to take into account Albert Einstein's point of view: "Insanity: doing the same thing over and over again and expecting different results".

The phenomenon of dark matter and dark energy can be quite explicable in terms of the hypothesis of the hidden Multiverse [35] [36] [37] [38]:

- invisibility of dark matter and dark energy is explained by mutual invisibility of parallel universes of the hidden Multiverse;

- failure to detect any of well-known chemical elements in composition of dark matter and dark energy is explained by inability to analyze contents of other universes located in other dimensions by tools we use on Earth;

- dark matter is other parallel universes of the hidden Multiverse adjacent to our universe;

- dark energy is other parallel universes of the hidden Multiverse, besides our universe and universes adjacent to it.

\section{Analysis of Data of WMAP and Planck Spacecrafts}

This structure of the hidden Multiverse can be defined more exactly due to data obtained by WMAP [84] and Planck [85] spacecrafts. Thus, according to WMAP data, our universe (in fact, our hidden Multiverse) is composed of $4.6 \%$ baryonic matter, $22.4 \%$ dark matter and $73.0 \%$ dark energy. And according to later measurements of the Planck spacecraft, our universe (again, in fact, our hidden Multiverse) is composed of $4.9 \%$ baryonic matter, $26.8 \%$ dark matter and $68.3 \%$ dark energy.

Therefore, believing that mass-energy of parallel universes has been substantially averaged over billions of years due to the existence of portals, i.e. their mass-energy can be accurately assumed to be equal, we find:

- the total number of parallel universes in the hidden Multiverse, which is $100 \% / 4.6 \%=21.7$ universes according to WMAP data and

$100 \% / 4.9 \%=20.4$ universes according to Planck data, i.e. $20 \ldots 22$ universes;

- the number of parallel universes in dark matter, which is $22.4 \% / 4.6 \%=4.9$ universes according to WMAP data and $26.8 \% / 4.9 \%=5.5$ universes according to Planck data, i.e. 5 ... 6 universes;

- the number of parallel universes in dark energy, which is $73.0 \% / 4.6 \%=15.9$ universes according to WMAP data and $68.3 \% / 4.9 \%=13.9$ universes according to Planck data, i.e. 14 ... 16 universes.

Thus, dark matter and dark energy turned out to be invisible parallel universes of the hidden Multiverse, beside ours, rather than some new microstruc- 
tures of our visible universe, which are currently looked for at the Large Hadron Collider. In other words, these are just other names of these universes.

Apparently, the foregoing does not fully correspond to assertions generally accepted in relativistic physics and astrophysics. Sir Isaac Newton wrote: "No great discovery was ever made without a bold guess". The same opinion was held by Niels Henrik David Bohr who said his catchphrase: “There is no doubt we have faced a mad theory. But the question is this. Is it really crazy enough to be right?"

However, the results of WMAP and Planck data analysis that give such evident explanation do not correspond to the probable structures of the hidden Multiverse shown in Figures 2-4. In these structures, tardyon universes cannot be adjacent to five-six tachyon universes and antiverses, since there is no space for two-three infinitely large parallel universes in the same dimension with the coordinate $q$.

Consequently, the structures of the hidden Multiverse shown in Figures 2-4 do not correspond to the WMAP and Planck data. And their fallacy is explained by the fact that they correspond only to the simplest case of implementation of the hidden Multiverse, which has only one extra dimension, i.e. they correspond to the principle of physical reality of complex numbers containing only one imaginary unit, for which formulas (4), (5) and (6) are right.

However, as follows from the WMAP and Planck data, implementation of the structures of the hidden Multiverse corresponding to calculation result obtained requires three extra dimensions. That is, formulas (4), (5), and (6) shall be corrected in accordance with the principle of physical reality of quaternions $\sigma+i_{1} \omega_{1}+i_{2} \omega_{2}+i_{3} \omega_{3}$ [86], containing three imaginary units $i_{1}, i_{2}, i_{3}$ connected by relations

$$
\begin{gathered}
i_{1}^{2}=i_{2}^{2}=i_{3}^{2}=1 \\
i_{1} i_{2} i_{3}=i_{2} i_{3} i_{1}=i_{3} i_{1} i_{2}=-1 \\
i_{1} i_{3} i_{2}=i_{2} i_{1} i_{3}=i_{3} i_{2} i_{1}=1
\end{gathered}
$$

In this case the corrected relativistic formulas will be as follows

$$
\begin{aligned}
m & =\frac{m_{0}\left(i_{1}\right)^{q}\left(i_{2}\right)^{r}\left(i_{3}\right)^{s}}{\sqrt{1-[v / c-(q+r+s)]^{2}}}=\frac{m_{0}\left(i_{1}\right)^{q}\left(i_{2}\right)^{r}\left(i_{3}\right)^{s}}{\sqrt{1-(w / c)^{2}}} \\
\Delta t & =\Delta t_{0}\left(i_{1}\right)^{q}\left(i_{2}\right)^{r}\left(i_{3}\right)^{s} \sqrt{1-[v / c-(q+r+s)]^{2}} \\
& =\Delta t_{0}\left(i_{1}\right)^{q}\left(i_{2}\right)^{r}\left(i_{3}\right)^{s} \sqrt{1-(w / c)^{2}} \\
l & =l_{0}\left(i_{1}\right)^{q}\left(i_{2}\right)^{r}\left(i_{3}\right)^{s} \sqrt{1-[v / c-(q+r+s)]^{2}} \\
& =l_{0}\left(i_{1}\right)^{q}\left(i_{2}\right)^{r}\left(i_{3}\right)^{s} \sqrt{1-(w / c)^{2}}
\end{aligned}
$$

where $q$ is the total number of parallel universes, penetration into which is made through portals, corresponding to the imaginary unit $i_{1}$, with increasing distance from our tardyon universe; 
$r$ is the total number of parallel universes, penetration into which is made through portals, corresponding to the imaginary unit $i_{2}$, with increasing distance from our tardyon universe;

$s$ is the total number of parallel universes, penetration into which is made through portals, corresponding to the imaginary unit $i_{3}$, with increasing distance from our tardyon universe;

$v$ is the velocity measured from our tardyon universe, which, therefore, can be called tardyon velocity;

$c$ is the speed of light;

$w=v-(q+r+s) c$ is the local velocity for corresponding universe, which can take values only in the range $0 \leq w<c$.

The structure of the hidden Multiverse corresponding to the formulas (10), (11) and (12) can be as in Figure 5. As can be seen, this structure is distinguished from the structure shown in Figure 4 by the fact that it contains three tachyon universes $i_{1}, i_{2}, i_{3}$ and three tachyon antiverses $i_{1}, i_{2}, i_{3}$, which provides required three extra dimensions. Thus, six-dimensional space of the hidden Multiverse has actually three extra dimensions $q, r, s$ including parallel universes, and three dimensions $x, y, z$ including material contents of each of these universes. So, space of the hidden Multiverse [87] is described by the formula $f_{q, r . s}(x, y, z)+i_{1} q+i_{2} r+i_{3} s$ where the function $f_{q, r, s}(x, y, z)$ describes

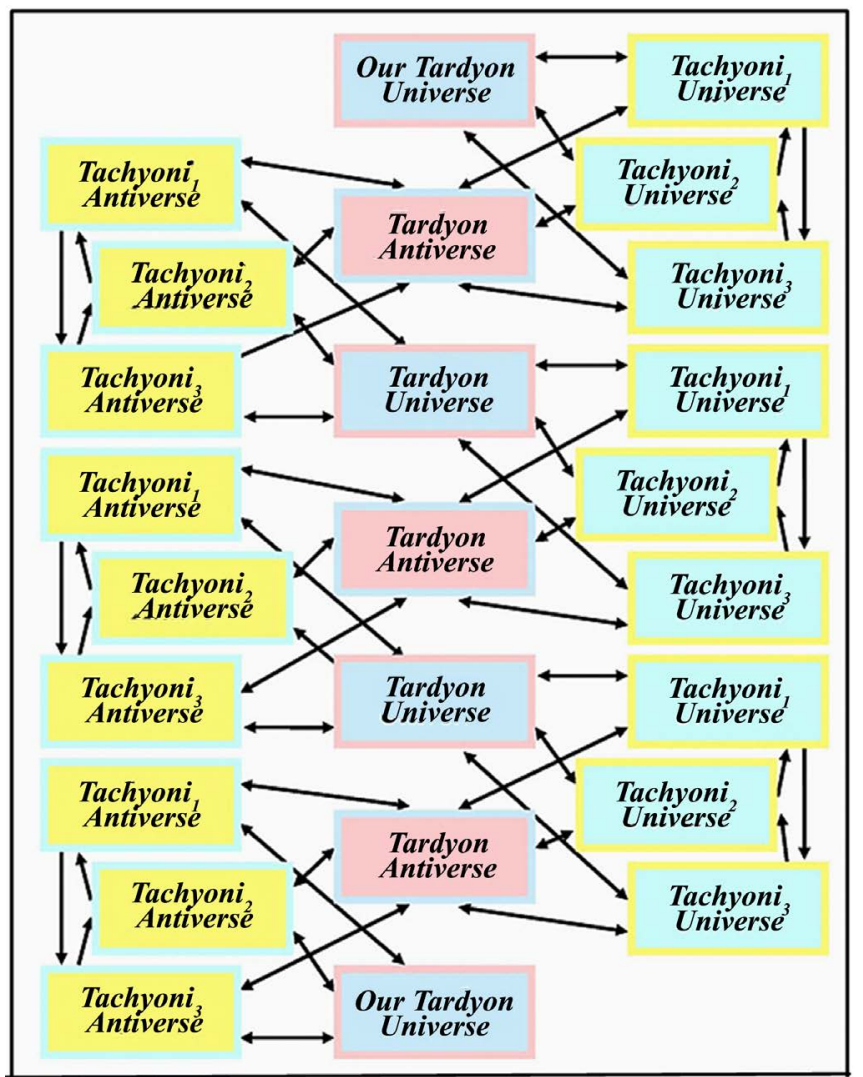

Figure 5. Structure of the hidden Multiverse corresponding to the principle of physical reality of quaternions. 
distribution in the coordinates $x, y, z$ of material contents in the corresponding parallel universe with coordinates $q, r, s$.

Lisa Randall wrote in this regard: "maybe we live in a three-dimensional spatial slit of a multidimensional universe".

Besides, the quaternion structure is distinguished by presence in it of both bidirectional portals corresponding to the formula (7) and unidirectional portals corresponding to formulas (8) and (9). It means that there is a danger to get into a unidirectional portal while travelling in the hidden Multiverse and never return back therefrom.

However, this structure of the hidden Multiverse also does not correspond to the results of WMAP and Planck data analysis, since it contains twenty-four parallel universes, rather than twenty to twenty two parallel universes. As explained above, this proves that our hidden Multiverse is connected to other Multiverses through corresponding portals and together with them forms a Hyperverse. Therefore, it can be argued that other invisible Multiverses of the Hyperverse, beside ours, form a dark space. In other words, the Hyperverse is a multidimensional (and possibly infinite-dimensional) Universum ${ }^{17}$ containing a very large number of Multiverses.

Besides, our hidden Multiverse can be connected to other Multiverses in various ways. Firstly, it can be connected to a different number of other Multiverses. Secondly, it can be connected to other Multiverses in various ways. Some probable variants of connection are shown in Figures 6-8. And finally, it can be connected to other Multiverses which have different structures and number of parallel universes. Herewith, there is a possibility that it is connected to other Multiverses corresponding to the principle of physical reality of various complex and hypercomplex numbers.

Figure 6 shows a structure diagram of our hidden Multiverse containing twenty-two parallel universes and connected twice to the same Multiverse of dark space, instead of its two missing parallel universes. In this structure diagram our tardyon universe is connected through corresponding portals to five adjacent parallel universes: two tachyon universes $i_{1}, i_{2}$ and three tachyon antiverses $i_{1}, i_{2}, i_{3}$.

Figure 7 shows another structure diagram of our hidden Multiverse containing twenty-one parallel universes and connected to three different Multiverses of dark space, instead of its three missing parallel universes. In this structure diagram our tardyon universe is connected through corresponding portals to five adjacent parallel universes: two tachyon universes $i_{1}, i_{2}$ and three tachyon antiverses $i_{1}, i_{2}, i_{3}$.

Figure 8 shows one more structure diagram of our hidden Multiverse containing twenty parallel universes and connected to three different Multiverses of dark space, instead of its four missing parallel universes. In this structure diagram our tardyon universe is connected through corresponding portals to six ${ }^{17}$ It is similar to a large city, in which our three-dimensional visible universe is just one of apartments. 


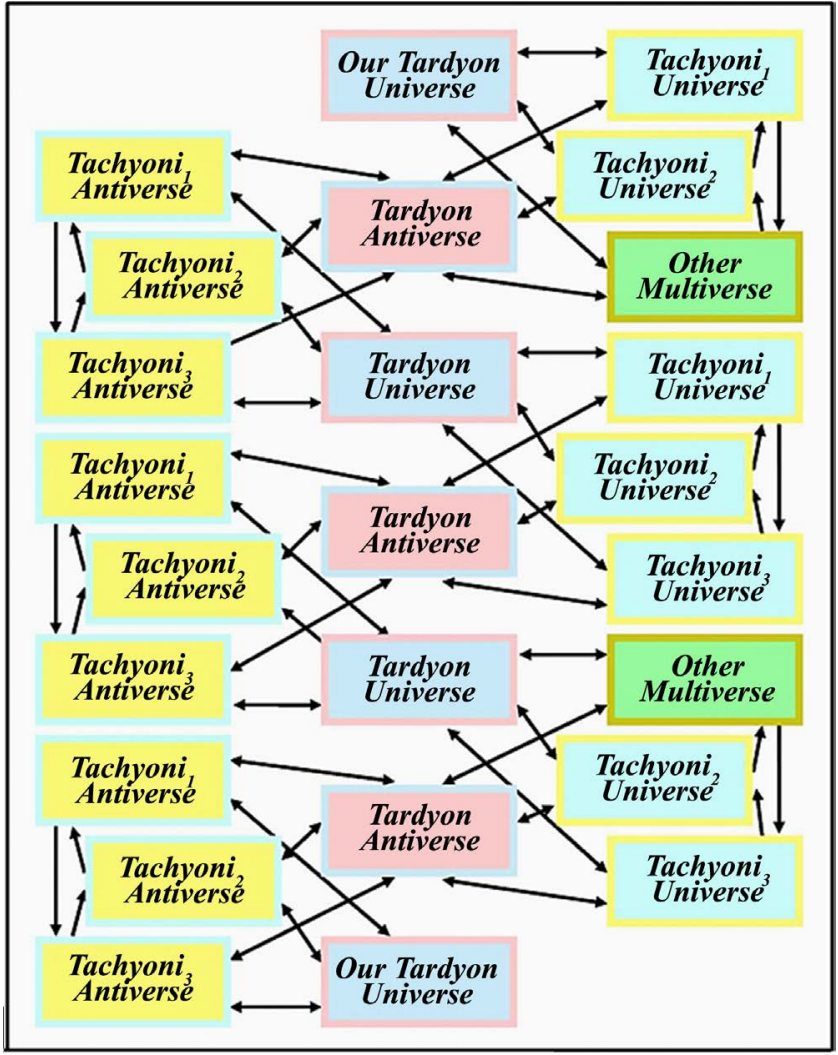

Figure 6. Quaternion structure of the hidden Multiverse.

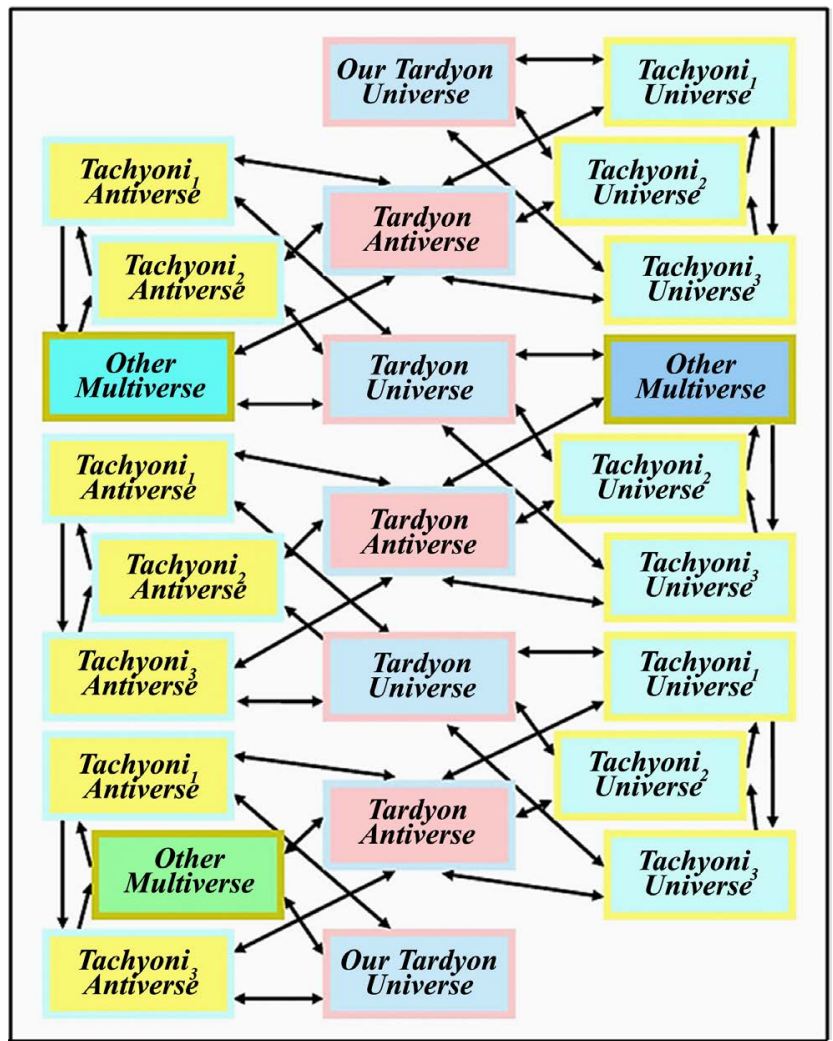

Figure 7. Another quaternion structure of the hidden Multiverse. 


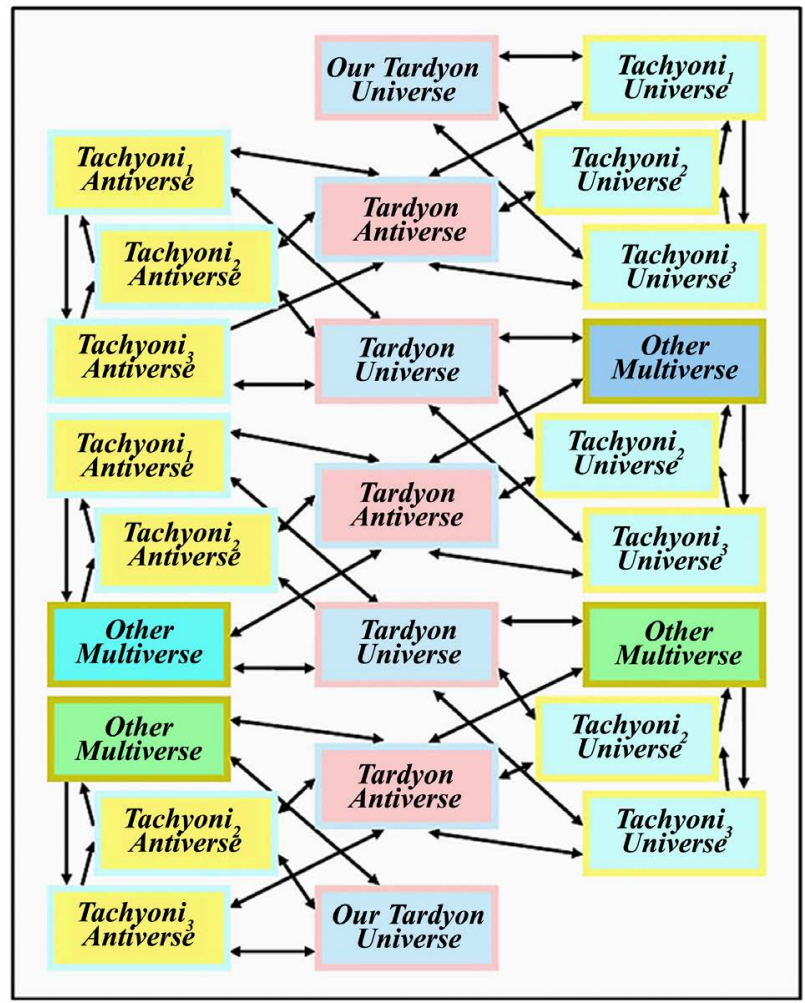

Figure 8. One more quaternion structure of the hidden Multiverse.

adjacent parallel universes: three tachyon universes $i_{1}, i_{2}, i_{3}$ and three tachyon antiverses $i_{1}, i_{2}, i_{3}$.

\section{Discovery of Dark Space}

There can be a lot of similar examples of structures of the hidden Multiverse provided. However, we are not currently able to say which of them actually exists, as we have not got enough experimental data. So, to answer this question we need additional astrophysical studies, including those aimed at experimental confirmation of dark space existence.

And this is presumably possible, although the above-mentioned phenomenon of dark space is just as invisible and unlearned, as the phenomenon of dark matter and dark space. Moreover, in contrast to the phenomenon of dark matter and dark space, it turned out to be undetectable by its physical manifestations. Therefore, it hasn't been discovered until very recently. It has been detected only due to mathematical processing of data obtained by the WMAP and Planck spacecrafts [17] [39], aimed at checking them for compliance with hypotheses of the hidden Multiverse and Hyperverse. However, since the structure of the Hyperverse includes the hidden Multiverse, in which our tardyon universe can have not only six (as in Figure 9(a)), but also five (as in Figure 9(b)) adjacent parallel universes, there can be portals on Earth to the universes of dark space. Therefore, existence of dark space can be confirmed by geophysical studies of portals and afterportal spaces and with the help of astrophysical studies. 


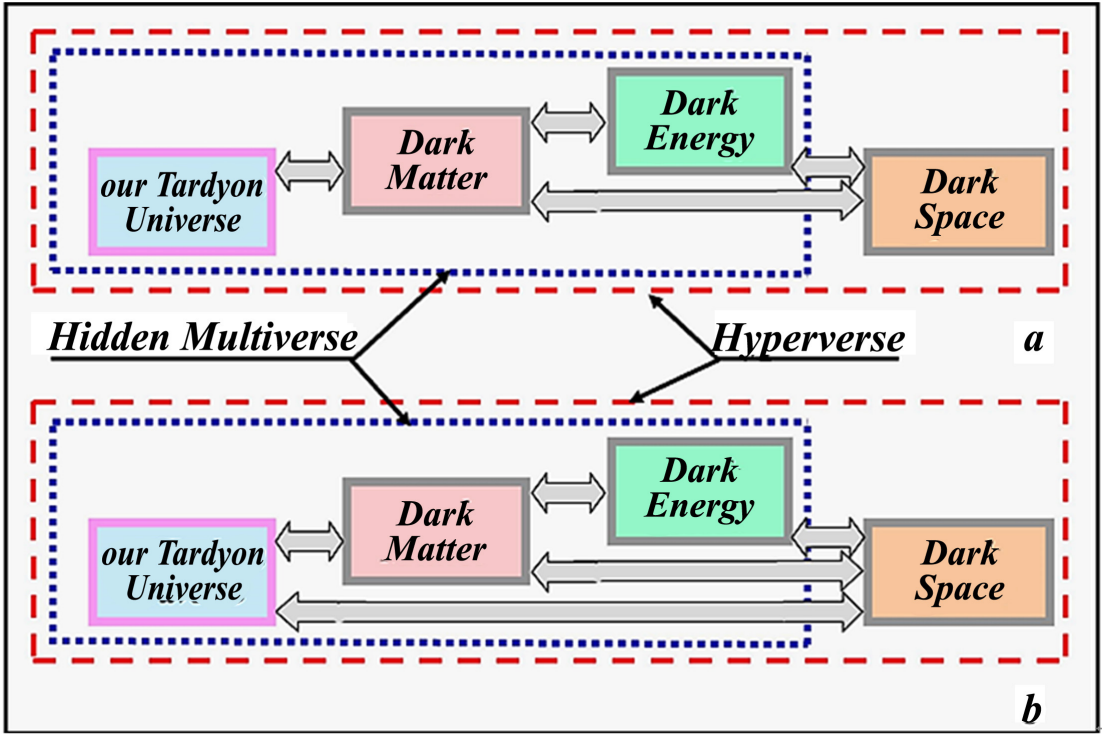

Figure 9. Structure of the Hyperverse.

\section{Verifiability of the Hypothesis of the Hidden Multiverse}

A large number of interesting hypotheses of Multiverse have been to date proposed, all of which are, however, unverifiable. Nevertheless, the proposed hypothesis of the hidden Multiverse is verifiable, since:

- the phenomenon of dark matter and dark energy, as is shown above, is its experimental confirmation. It is also experimentally confirmed by mass defect that is often detected in experiments at the Large Hadron Collider, i.e. a situation in which the total mass of subatomic particles turned out to be greater before acceleration than their total mass near the point of astrophysical singularity $v \rightarrow c$. This situation can be explained by formation of tachyons and their transition to adjacent tachyon universes and antiverses through micro portals.

Although these two statements logically follow from the foregoing and correspond to common sense, they raise some doubts, since they do not presumably exclude other explanations of the mentioned experimental data. However, the third proof of existence of invisible universes given below leaves no doubt whatsoever. The point of this proof is as follows. Since stars are placed differently in invisible parallel universes adjacent to our visible universe, star maps in areas beyond the portals might be other than those observed from Earth. Therefore, as we move from our universe to one of the adjacent universes through portals, which are the so-called anomalous zones [88], star maps of our visible universe are gradually replaced with star maps corresponding to adjacent universes that are not visible from Earth. And even given shallow penetration into portals such differences would likely be more noticeable than in astronomical observations of Sir Arthur Stanley Eddington in 1919 that confirmed the deviation of light rays near the Sun, predicted by the general theory of relativity. Thus, in order to make sure that universes unobservable from Earth exist, one should take a pic- 
ture of starry sky in portals, which are numerous on Earth, and define differences comparing these pictures with those taken in observatories. Therefore, the discovery of the invisible universes in the proposed way seems quite convincing and expected in the near future.

Consequently, the hypothesis of the hidden Multiverse considered has every reason to be called a theory.

\section{Dark Dimensions}

However, one should not think that other physical worlds are just other parallel universes. As proved above, all concrete imaginary numbers are physically real. And physical entities corresponding to them also form their parallel worlds.

For example, in the Euler formula $\exp (i x)=\cos x+i \sin x$, describing oscillatory processes of any physical nature, such as mechanical, hydraulic, acoustic, electromagnetic, etc., both summands of its right part are physically real. However, no one can currently say what the component $i \sin x$ is, if the component $\cos x$ corresponds to mechanical oscillations of a pendulum. It's the same as in the case when the component $\cos x$ describes other oscillatory processes, or when concrete imaginary numbers describe any physical objects and processes described by other formulas. Special research is needed for each specific case, like that conducted earlier when clarifying physical nature of imaginary reactances in the theory of electric circuits or physical nature of relativistic formulas at superluminal speeds in the STR. Such research will allow us to learn a structure of contents of parallel universes and relationship between contents of our and other universes. However, this is a task of future.

In the meantime, we just note that any concrete numbers, both real and imaginary, always correspond to the results of measurements. Therefore, concrete imaginary numbers shall be called dark dimensions by analogy with dark matter and dark energy, since they are still absolutely inexplicable and objects of measurements corresponding to them are absolutely invisible.

\section{Conclusions}

The monograph has proved that dark matter and dark energy discovered in the previous century still seem completely inexplicable only because their explanation is not sought where it is. Everybody looks for the explanation exclusively in our visible Monoverse, the hypothesis of which follows from the principle of light speed non-exceedance postulated in the STR. The hypothesis of the Monoverse supposes that there seems to be nowhere to look for the explanation of the phenomenon of dark matter and dark energy.

The principle of light speed non-exceedance in the STR turned out to be in demand, because all its relativistic formulas in this theory were inexplicable. They couldn't be explained, as they implied that mass, time, distance and other physical quantities at superluminal speeds took values measured by imaginary numbers. Creators of the STR did not know what this meant, and therefore 
could not explain their theory. Therefore, they needed this principle in order to avoid the necessity to recognize it.

However, this postulate didn't convince other scientists, who were more interested in scientific truth than prestigious considerations. In addition, some physical discoveries, for example, Cherenkov radiation produced by charged particles moving faster than light, could cause such doubts. Therefore, it was quite natural to search for other physical phenomena that could disprove the principle of light speed non-exceedance.

As a result, the OPERA collaboration attempted to refute the postulate on light speed non-exceedance by proving the existence of superluminal neutrinos. On September 22, 2011 a sensational report on successful completion of this very complex experiment was published. However, in half a year the OPERA experiment was refuted by the ICARUS experiment. Therefore, the question of existence of superluminal neutrinos and physical reality of imaginary numbers remained open.

However, in 2008-2010, i.e. before completion of the OPERA experiment, results of other experiments were published. They successfully proved physical reality of concrete imaginary numbers, and therefore made the OPERA experiment unnecessary. These and subsequent publications on experimental studies of oscillatory processes in linear electric circuits have also refuted the principle of light speed non-exceedance, which is just an unproved assumption.

Therefore, the conclusion about existence of the Monoverse derived from the principle of light speed non-exceedance in the STR also turned out to be wrong. Until recently an explanation for dark matter and dark energy was unsuccessfully sought in the Monoverse. However, they weren't found there. The corrected relativistic formulas of the STR have allowed creating a verified hypothesis of the hidden Multiverse. Existence of invisible parallel universes therein has explained the phenomenon of dark matter and dark energy. Moreover, existence of other Multiverses outside the hidden Multiverse has also discovered and explained a previously unknown phenomenon of dark space. It has been shown that our visible universe together with invisible parallel universes of dark matter, dark energy and dark space form the Hyperverse.

The structure of the hidden Multiverse has been clarified in the course of mathematical analysis of experimental data obtained by the WMAP and Planck spacecrafts. Their mathematical processing has made it possible to determine that the hidden Multiverse has a quaternion structure in six-dimensional space and contains twenty-twenty-two parallel universes. Of them, five-six invisible parallel universes of dark matter are adjacent to our visible universe, and the remaining invisible parallel universes of dark energy in the hidden Multiverse are further away from our visible universe. These universes are interconnected by unidirectional and bidirectional portals. And some portals connect them with invisible parallel universes of the Hyperverse, which are outside the hidden Multiverse. 
In this book, there are naturally no answers to many other issues of astrophysics. But they cannot but be, since each new theory giving answers to questions posed by previous theories in turn inevitably gives rise to new questions. And the authors of other even more new theories and hypotheses are to answer them. This is the inevitable logic of science development.

Nevertheless, the main features of dark matter and dark energy-why they are invisible and why no molecules, atoms and subatomic particles are found in them-are clearly and convincingly explained in section 6. And in section 9 it is explained how the existence of invisible universes, by which the phenomenon of dark matter and dark energy is generated, can be proved in the most indisputable way with the help of photographs of the starry sky in the portals.

However, imaginary numbers are used not only in the theory of electric circuits, the special theory of relativity and astrophysics. They are also used in all other exact sciences. Imaginary physical quantities corresponding to them are called dark dimensions. Learning of their physical nature would require additional studies, which would largely determine the content of future science.

\section{Conflicts of Interest}

The author declares no conflict interest regarding the publication of this paper.

\section{References}

[1] Antonov, A.A. (2008) European Journal of Scientific Research, 21, 627-641.

[2] Antonov, A.A. (2009) European Journal of Scientific Research, 28, 193-204.

[3] Antonov, A.A. (2010) American Journal of Scientific and Industrial Research, 1, 342-349. https://doi.org/10.5251/ajsir.2010.1.2.342.349

[4] Antonov, A.A. (2010) International Journal of Pure and Applied Sciences and Technology, 1, 1-12.

[5] Antonov, A.A. (2010) General Mathematics Notes, 1, 11-16. https://doi.org/10.17686/sced_rusnauka_2010-887

[6] Antonov, A.A. (2013) International Journal of Management, IT and Engineering, 3, 219-230.

[7] Antonov, A.A. (2014) American Journal of Scientific and Industrial Research, 5, 40-52.

[8] Antonov, A.A. (2015) Journal of Russian Physical and Chemical Society, 87, 328-355. (In Russian)

[9] Antonov, A.A. (2015) General Mathematics Notes, 31, 34-53. http://www.emis.de/journals/GMN/yahoo_site_admin/assets/docs/4_GMN-9212-V 31N2.1293701.pdf

[10] Antonov, A.A. (2015) American Journal of Electrical and Electronics Engineering, 3, 124-129.

[11] Antonov, A.A. (2015) Global Journal of Physics, 2, 145-149. http://gpcpublishing.com/index.php?journal=gjp\&page=article\&op=view\&path\%5 $\underline{\mathrm{B} \% 5 \mathrm{D}=294 \& \text { path } \% 5 \mathrm{~B} \% 5 \mathrm{D}=\mathrm{pdf} \_14}$

[12] Antonov, A.A. (2016) PONTE, 72, 131-142. 
https://doi.org/10.21506/j.ponte.2016.7.9

[13] Antonov, A.A. (2016) Journal of Modern Physics, 7, 2299-2313. https://doi.org/10.4236/jmp.2016.716198

[14] Antonov, A.A. (2016) International Review of Physics, 10, 31-35. https://www.Praiseworthyprize.org/jsm/index.php?journal=irephy\&page=article\&o $\mathrm{p}=$ view\&path\%5B\%5D=18615

[15] Antonov, A.A. (2016) General Mathematics Notes, 35, 40-63. http://www.geman.in/yahoo_site_admin/assets/docs/4_GMN-10932-V35N2.318951 46.pdf

[16] Antonov, A.A. (2017) Norwegian Journal of Development of the International Science, 6, 50-63. http://www.njd-iscience.com

[17] Antonov, A.A. (2018) Natural Science, 10, 11-30. https://doi.org/10.4236/ns.2018.101002

[18] Antonov, A.A. (2014) Global Journal of Science Frontier Research: A Physics \& Space Science, 14, 51-59.

https://globaljournals.org/GJSFR_Volume14/7-Verification-of-the-Second-Postulat e.pdf

[19] Antonov, A.A. (2011) British Journal of Science, 2, 51-60. https://doi.org/10.17686/sced_rusnauka_2011-892

[20] Antonov, A.A. (2012) Encyclopedia of Russian Thought. Reports to Russian Physical Society, 16, 3-20.

[21] Antonov, A.A. (2012) International Journal of Pure and Applied Sciences and Technology, 12, 43-56.

[22] Antonov, A.A. (2015) International Journal of Advanced Research in Physical Science, 2, 25-32.

[23] Antonov, A.A. (2015) American Journal of Modern Physics, 4, 1-9. https://doi.org/10.11648/j.ajmp.20150401.11

[24] Antonov, A.A. (2015) International Journal of Physics, 3, 84-87.

[25] Antonov, A.A. (2015) Cosmology, 19, 40-61.

[26] Antonov, A.A. (2015) Optics, 4, 43-47.

[27] Antonov, A.A. (2015) American Journal of Modern Physics, 4, 180-188. https://doi.org/10.11648/j.ajmp.20150404.14

[28] Antonov, A.A. (2016) Frontiers of Astronomy, Astrophysics and Cosmology, 2, 1-9.

[29] Antonov, A.A. (2016) Journal of Modern Physics, 7, 1228-1246. https://doi.org/10.4236/jmp.2016.710111

[30] Antonov, A.A. (2016) American Journal of Modern Physics. http://www.sciencepublishinggroup.com/news/sciencepgfrontiersinfo?articleid=93

[31] Antonov, A.A. (2016) Journal of Modern Physics, 7, 1933-1943. https://doi.org/10.4236/jmp.2016.714170

[32] Antonov, A.A. (2016) Global Journal of Science Frontier Research: A Physics and Space Science, 16, 4-12.

[33] Antonov, A.A. (2017) Global Journal of Science Frontier Research. http://blog.gjsfr.org/2017/02/verifiable-hidden-multiverse.html

[34] Antonov, A.A. (2017) Natural Science, 9, 43-62. https://doi.org/10.4236/ns.2017.93005

[35] Antonov, A.A. (2015) Journal of the Russian Physical and Chemical Society, 87, 
63-76. (In Russian) http://www.rusphysics.ru/files/Antonov.Obsor.pdf

[36] Antonov, A.A. (2016) Global Journal of Science Frontier Research, 15. http://blog.gjsfr.org/2016/09/explanation-of-dark-matter-and-dark.html

[37] Antonov, A.A. (2017) Journal of Modern Physics, 8, 567-582. https://doi.org/10.4236/jmp.2017.84038

[38] Antonov, A.A. (2016) PONTE, 72, 288-300. https://doi.org/10.21506/j.ponte.2016.9.22

[39] Antonov, A.A. (2018) Journal of Modern Physics, 9, 14-34. https://doi.org/10.4236/jmp.2018.91002

[40] Ohm, G.S. (1826) Journal fur Chemie und Physik, 46, 137-166.

[41] Steinmetz, C.P. (2010) Theory and Calculation of Electric Circuit. Nabu Press, Charleston.

[42] Larmor, J.J. (1897) Philosophical Transactions of the Royal Society A: Mathematical, Physical and Engineering Sciences, 190, 205-300. https://doi.org/10.1098/rsta.1897.0020

[43] Lorentz, H.A. (1899) Proceeding of the Royal Netherlands Academy of Arts and Science, 1, 427-442.

[44] Poincaré, H. (1905) Comptes Rendus, 140, 1504-1508.

[45] Einstein, A. (1905) Annalen der Physik, 17, 891-921. https://doi.org/10.1002/andp.19053221004

[46] Einstein, A. (1920) Relativity: The Special and General Theory. H. Holt and Company, New York.

[47] Bohm, D. (2006) The Special Theory of Relativity. Routledge, Abingdon-on-Thames.

[48] Hawking, S.W. and Penrose, R. (2010) The Nature of Space and Time. Princeton University Press, Princeton. https://doi.org/10.1515/9781400834747

[49] Tanaka, S. (1960) Progress of Theoretical Physics (Kyoto), 24, 171-200. https://doi.org/10.1143/PTP.24.171

[50] Terletsky, Y.P. (1966) Paradoxes of the Relativity Theory. Nauka Publishing, Moscow. (In Russian)

[51] Feinberg, G. (1967) Physical Review, 159, 1089-1105. https://doi.org/10.1103/PhysRev.159.1089

[52] Bilaniuk, O.-M.P. and Sudarshan, E.C.G. (1969) Physics Today, 22, 43-51. https://doi.org/10.1063/1.3035574

[53] Recami, E., Fontana, F. and Garavaglia, R. (2000) International Journal of Modern Physics A, 15, 2793-2812. https://doi.org/10.1142/S0217751X00001403

[54] Hill, J.M. and Cox, B.J. (2012) Proceeding of Royal Society A: Mathematical, Physical and Engineering Sciences, 468, 4174-4192. https://doi.org/10.1098/rspa.2012.0340

[55] Antonov, A.A. (2015) Where to Look for Alien Civilisations, Cosmology. Commentaries: Stephen Hawking's Aliens. The Search for Intelligent Extraterrestrial Life. Project Breakthrough Listen. http://cosmology.com/Aliens1.html

[56] Antonov, A.A. (2016) How Portals of the Invisible Multiverse Operate. Science PG Frontiers.

http://www.sciencepublishinggroup.com/news/sciencepgfrontiersinfo?articleid=147

[57] Antonov, A.A. (2016) Philosophy and Cosmology, 6, 11-27. 
http://ispcjournal.org/journals/2016-16/Antonov16.pdf

[58] Alfvén, H. (1966) Worlds-Antiworlds: Antimatter in Cosmology. W. H. Freeman \& Co., San Francisco.

[59] Foot, R. (2002) Shadowlands: Quest for Mirror Matter in the Universe. Universal Publishers, Parkland.

[60] Frazer, G. (2004) Antimatter: The Ultimate Mirror. Cambridge University Press, Cambridge.

[61] Santilli, R.M. (2006) Isodual Theory of Antimatter: With Applications to Antigravity, Grand Unification and Cosmology. Springer Netherlands, Dordrecht.

[62] Mazure, A. and Le Brun, V. (2012) Matter, Dark Matter, and Anti-Matter: In Search of the Hidden Universe. Springer-Verlag, Berlin. https://doi.org/10.1007/978-1-4419-8822-5

[63] Freeman, K. and McNamara, G. (2006) In Search of Dark Matter. Springer, Berlin.

[64] Nicolson, I. (2007) Dark Side of the Universe: Dark Matter, Dark Energy, and the Fate of the Cosmos. Johns Hopkins University Press, Baltimore.

[65] Ruiz-Lapuente, P. (2010) Dark Energy: Observational and Theoretical Approaches. Cambridge University Press, Cambridge. https://doi.org/10.1017/CBO9781139193627

[66] Amendola, L. and Tsujikawa, S. (2010) Dark Energy: Theory and Observations. Cambridge University Press, Cambridge. https://doi.org/10.1017/CBO9780511750823

[67] Bertone, G. (2013) Particle Dark Matter: Observations, Models and Searches. Cambridge University Press, Cambridge.

[68] Sanders, R.H. (2014) The Dark Matter Problem: A Historical Perspective. Cambridge University Press, Cambridge.

[69] deGrasse, T.N. (2017) Astrophysics for People in a Hurry. W. W. Norton \& Company, London.

[70] Oort, J.H. (1932) Bulletin of the Astronomical Institutes of the Netherlands, 6, 249-287. http://adsabs.harvard.edu/abs/1932BAN.....6..249O

[71] Zwicky, F. (1933) Helvetica Physica Acta, 6, 110-127. http://adsabs.harvard.edu/abs/1933AcHPh...6..110Z

[72] Perlmutter, S. (2012) Reviews of Modern Physics, 84, 1127-1149. https://doi.org/10.1103/RevModPhys.84.1127

[73] Schmidt, B.P. (2012) Reviews of Modern Physics, 84, 1151-1163. https://doi.org/10.1103/RevModPhys.84.1151

[74] Riess, A.G. (2012) Reviews of Modern Physics, 84, 1165-1175. https://doi.org/10.1103/RevModPhys.84.1165

[75] Kaku, M. (2005) Parallel Worlds: A Journey through Creation, Higher Dimensions, and the Future of the Cosmos. Doubleday, New York.

[76] Deutch, D. (1998) The Fabric of Reality: The Science of Parallel Universes and Its Implications. Penguin Books, New York.

[77] Greene, B. (2005) The Fabric of the Cosmos: Space, Time, and the Texture of Reality. Penguin Books, London.

[78] Vilenkin, A. (2007) Many Worlds in One: The Search for Other Universes. Hill and Wong, New York.

[79] Carr, B. (2009) Universe or Multiverse? Cambridge University Press, Cambridge. 
[80] Gribbin, J. (2010) In Search of the Multiverse: Parallel Worlds, Hidden Dimensions, and the Ultimate Quest for the Frontiers of Reality. Wiley \& Sons Inc., Hoboken.

[81] Greene, B. (2011) The Hidden Reality: Parallel Universes and the Deep Laws of the Cosmos. Vintage, New York.

[82] Hawking, S. and Mlodinow, L. (2012) The Grand Design. Bantam, New York.

[83] Tegmark, M. (2014) Our Mathematical Universe: My Quest for the Ultimate Nature of Reality. Vintage, New York.

[84] Hinshaw, G., Larson, D., Komatsu, E., Spergel, D.N., Bennett, C.L., Dunlley, J., et al. (2013) Nine Year Wilkinson Anisotropy Probe (WMAP) Observations: Cosmological Parameter Results.

[85] Adam, R., Ade, P.A.R., Aghanim, N., Akrami, Y., Alves, M.I.R., Argũeso, F., et al. (2015) Plank 2015 Results. 1. Overview of Products and Scientific Results.

[86] Kantor, I.L. and Solodovnikov, A.S. (1989) Hypercomplex Numbers. Springer Verlag, Berlin. https://doi.org/10.1007/978-1-4612-3650-4

[87] Antonov, A.A. (2015) Global Journal of Science Frontier Research A: Physics and Space Science, 15, 8-15.

https://globaljournals.org/GJSFR_Volume15/2-Quaternion-Structure-of-the-Hidde $\underline{\text { n.pdf }}$

[88] Chernobrov, V.A. (2000) Encyclopaedia of Mysterious Places of the Earth. Veche Publishing, Bucharest. (In Russian) 